\title{
P-Store: Genuine Partial Replication in Wide Area Networks
}

\author{
Nicolas Schiper \\ University of Lugano \\ 6904 Lugano, Switzerland \\ nicolas.schiper@usi.ch
}

\author{
Pierre Sutra \\ Université Paris VI and INRIA Rocquencourt \\ 75016 Paris, France \\ pierre.sutra@lip6.fr
}

\author{
Fernando Pedone \\ University of Lugano \\ 6904 Lugano, Switzerland \\ fernando.pedone@usi.ch
}

\begin{abstract}
Partial replication is a way to increase the scalability of replicated systems: updates only need to be applied to a subset of the system's sites, thus allowing replicas to handle independent parts of the workload in parallel. In this paper, we propose P-Store, a partially replicated key-value store for wide area networks. In P-Store, each transaction $T$ optimistically executes on one or more sites and is then certified to guarantee serializability of the execution. The certification protocol is genuine, it only involves sites that replicate data items read or written by $T$, and incorporates a mechanism to minimize a convoy effect. P-Store makes a thrifty use of an atomic multicast service to guarantee correctness: no messages need to be multicast during $T$ 's execution and a single message is multicast to certify $T$. In case $T$ is global, that is, $T$ 's execution is distributed at different geographical locations, an extra vote phase is required. Our approach may offer better scalability than previously proposed solutions that either require multiple atomic multicast messages to execute $T$ or are non-genuine. Experimental evaluations reveal that the convoy effect plays an important role even when one percent of the transactions are global. We also compare the scalability of our approach to a fully replicated solution when the proportion of global transactions and the number of sites vary.
\end{abstract}

\section{INTRODUCTION}

By allowing sites to store a subset of the application data and split the load among replicas, partial replication improves the scalability of replicated systems. With partial replication, access locality is favored by replicating data close to clients, and storage resources are used sparingly.

In this paper, we present P-Store, a scalable distributed key-value store that supports partial replication and transparent transactional access. P-Store assumes a wide area network environment where sites are clustered in groups (e.g., data centers) and seeks to minimize costly and slow inter-group communication.

The solution we propose is flexible and scalable in a precise sense as we explain next. Data items may be replicated anywhere and any number of times provided that sites of a given group replicate the same set of data items. Transaction execution does not require data items to be accessed from the same site, allowing more flexibility when partitioning data. Read requests are executed optimistically with no inter-site synchronization; transactions are then certified to guarantee serializability of the execution. To improve scalability, the certification protocols we present ensure genuine partial replication:
- For any submitted transaction $T$, only database sites that replicate data items read or written by $T$ exchange messages to certify $T$.

In P-Store, correctness relies on the use of an atomic multicast service to order transactions that operate on the same data items. We make an economical use of this service: to execute and certify each transaction $T$, a single message is atomically multicast. In case $T$ is global, an extra vote phase consisting of a single round of message exchange is needed to ensure agreement on $T$ 's outcome.

This is in contrast to previously proposed solutions that either atomically multicast multiple messages to handle each transaction [1], [2] or are non-genuine [3], [4], [5]. To the best of our knowledge, this is the first partial database replication protocol that is genuine and uses a single atomic multicast message per transaction.

The first certification protocol we propose is simple but vulnerable to a convoy effect that slows down transaction certification due to global transactions, i.e., transactions that involve multiple groups. To mitigate this undesired phenomenon, we propose a second protocol that doubles the throughput of the first protocol even when only $1 \%$ of transactions are global-this advantage grows when the percentage of global transactions increases.

We further study the performance of P-Store and compare its scalability to a fully replicated solution when the percentage of global transactions and the number of groups vary. P-Store provides a linear scale-out up to eight groups and when a fourth of the transactions are global. With this number of groups, P-Store allows to almost double the peak throughput of the fully replicated scheme, and can process multiple thousands of update transactions per second when each data items is replicated three times and inter-group links have a delay of 50 milliseconds and 10 megabits per second of bandwidth. Preliminary experimental results suggest that partial replication is interesting in systems with four or more groups when global transactions access few groups.

The rest of the paper is structured as follows. Section II introduces our model and assumptions. Sections III and IV respectively present P-Store and the two certification protocols; the current state-of-the-art is surveyed in Section V. The implementation of P-Store is sketched in Section VI and empirical results are reported in Section VII. Section VIII 
concludes the paper. Due to space limitations, we only briefly discuss the correctness of P-Store in Section IV; the full proofs can be found in [6].

\section{System Model And Definitions}

\section{A. Sites, Groups, and Links}

We consider a system $\Pi=\left\{s_{1}, \ldots, s_{n}\right\}$ of sites, each equipped with a local database. Sites communicate through message passing and do not have access to a shared memory nor a global clock. We assume the benign crash-stop failure model: sites may fail by crashing, but do not behave maliciously. A site that never crashes is correct; otherwise it is faulty.

The system is asynchronous, i.e., messages may experience arbitrarily large (but finite) delays and there is no bound on relative site speeds. To circumvent the FLP impossibility result [7] and make atomic multicast implementable, we further assume that the system is augmented with unreliable failure detectors [8]. The exact failure detector needed depends on the atomic multicast algorithm. Hereafter, we assume an atomic multicast service, as defined in II-D.

We define $\Gamma=\left\{g_{1}, \ldots, g_{m}\right\}$ as the set of site groups in the system. Groups are disjoint, non-empty, and satisfy $\bigcup_{g \in \Gamma} g=\Pi$. For each site $s \in \Pi, \operatorname{group}(s)$ identifies the group $s$ belongs to. A group $g$ that contains at least one correct site is correct; otherwise $g$ is faulty.

Communication links are quasi-reliable, i.e., they do not create, corrupt, nor duplicate messages, and for any two correct sites $s$ and $s^{\prime}$, and any message $m$, if $s$ sends $m$ to $s^{\prime}$, then $s^{\prime}$ eventually receives $m$.

\section{B. Database and Replication Model}

A database $\mathcal{D}$ is a finite set of tuples $(k, v, t s)$, or data items, where $k$ is a key, $v$ its value, and $t s$ is its version number. Each site holds a partial copy of the database. For each site $s_{i}$, we denote by $\operatorname{Items}\left(s_{i}\right) \subseteq \mathcal{D}$ the set of keys replicated at site $s_{i}$. Given a site $s_{i}$ and a key $k$ replicated at site $s_{i}$, $\operatorname{Version}\left(k, s_{i}\right)$ returns the version of $k$ stored at $s_{i}$. We suppose that, initially, for every key $k$ and every site $s_{i}, \operatorname{Version}\left(k, s_{i}\right)=1$. We assume that sites in the same group replicate the same set of data items, that is, $\forall g \in \Gamma: \forall s, s^{\prime} \in g: \operatorname{Items}(s)=\operatorname{Items}\left(s^{\prime}\right)$, and we allow data items to be replicated in more than one group. For every key $k$ in the database, there exists a correct site $s_{i}$ that replicates the value associated with $k$, i.e., $k \in \operatorname{Items}\left(s_{i}\right)$.

A transaction is a sequence of read and write operations on data items followed by a commit or an abort operation. A read on some key $k$ by transaction $T$, denoted $r_{T}[k]$, returns the value associated with $k$ as well as its corresponding version. A write performed by $T$ is designated as $w_{T}[k, v, t s]$, where $v$ is the value written on key $k$, and $t s$ is its version. For simplicity, we hereafter represent a transaction $T$ as a tuple $(i d, r s, w s, u p)$ where $i d$ is the unique identifier of $T$, rs is the set of key-version pairs read by $T$, ws is the set of keys written by $T$, and up contains the updates of $T$. More precisely, $u p$ is a set of tuples $(k, v)$, where $v$ is the new value $T$ associates to $k$; the set T.ws equals $\{k:(k, v) \in T . u p\}$, where we refer to element $e$ of $T$ 's tuple as T.e.

For every transaction $T$, Items $(T)$ is the set of keys read or written by $T$. Transactions $T$ and $T^{\prime}$ read-write conflict if one transaction reads a key $k$ that the other transaction updates. We do not consider write-write conflicts because for each key $k$, the certification protocols we propose totally order updates to $k$. Every transaction $T$ is associated to a unique site: $\operatorname{Proxy}(T)$, which submits $T$ 's read and write requests on behalf of a client. We denote WReplicas $(T)$ as the set of sites that replicate at least one data item written by $T$, and $\operatorname{Replicas}(T)$ as the sites that replicate at least one data item read or written by $T$. Transaction $T$ is local iff for any site $s$ in $\operatorname{Replicas}(T)$, Items $(T) \subseteq \operatorname{Items}(s)$; otherwise, $T$ is global.

Knowledge about whether $T$ is global or local, Items $(T)$, WReplicas $(T)$, and Replicas $(T)$ is only needed at certification time, i.e., after $T$ 's execution. However, we require sites to know where data items are located.

\section{Data Consistency Criteria}

On each site, the local database ensures order-preserving serializability: the local execution of transactions has the same effect as a serial execution of these transactions in which the commit order is preserved [9]. This condition is typically met by relying on two-phase locking.

In this paper, we provide a partial replication protocol that ensures the transaction execution on multiple partial copies of the database is equivalent to some one-copy serial execution of the same set of transactions. More precisely, the devised protocol ensures one-copy serializability (1SR) [10].

\section{Atomic Multicast}

We assume that our system is equipped with an atomic multicast service that allows messages to be disseminated to any subset of groups in $\Gamma[11],[12]$. For every message $m$, m.dst denotes the groups to which $m$ is multicast. A message $m$ is multicast by invoking A-MCast $(m)$ and delivered with A-Deliver $(m)$. We define the relation $<$ on the set of messages sites A-Deliver as follows: $m<m^{\prime}$ iff there exists a site that A-Delivers $m$ before $m^{\prime}$.

Atomic multicast satisfies the following properties: (i) uniform integrity: for any site $s$ and any message $m, s$ ADelivers $m$ at most once, and only if $s \in m$.dst and $m$ was previously A-MCast, (ii) validity: if a correct site $s$ A-MCasts a message $m$, then eventually all correct sites $s^{\prime} \in$ m.dst A-Deliver $m$, (iii) uniform agreement: if a site $s$ A-Delivers a message $m$, then eventually all correct sites $s^{\prime} \in m$.dst A-Deliver $m$, (iv) uniform prefix order: for any two messages $m$ and $m^{\prime}$ and any two sites $s$ and $s^{\prime}$ such 
that $\left\{s, s^{\prime}\right\} \subseteq m$.dst $\cap m^{\prime}$.dst, if $s$ A-Delivers $m$ and $s^{\prime}$ A-Delivers $m^{\prime}$, then either $s$ A-Delivers $m^{\prime}$ before $m$ or $s^{\prime}$ A-Delivers $m$ before $m^{\prime}$, (v) uniform acyclic order: the relation $<$ is acyclic.

To guarantee genuine partial replication, we require atomic multicast protocols to be genuine [13]: an algorithm $\mathcal{A}$ solving atomic multicast is genuine iff for any admissible run $R$ of $\mathcal{A}$ and for any site $s$, in $R$, if $s$ sends or receives a message, then some message $m$ is A-MCast, and either $s$ is the site that A-MCasts $m$ or $s \in m$.dst.

\section{The Lifetime of A TRAnSACTION In P-STORE}

We present the lifetime of transactions in our partially replicated system $\mathrm{P}$-Store. We consider a transaction $T$ and comment on the different states $T$ can be in.

- Executing: Each read operation on key $k$ is executed at some site that stores $k ; k$ and the data item version $t s$ read are stored as a pair $(k, t s)$ in T.rs. Reads are optimistic, that is, no synchronization between sites in Replicas $(T)$ occurs to guarantee the consistency of $T$ 's view of the database; later on, when $T$ is in the Submitted state, a certification protocol checks that $T$ read the correct data item versions. Every update of key $k$ to some value $v$ is buffered as a pair $(k, v)$ in T.up. If a read is issued on a key $k$ that was previously updated by $T$, the corresponding value stored in T.up is returned.

When $\operatorname{Proxy}(T)$ requests a commit, $T$ passes to the Committed state if $T$ is read-only and local. Otherwise, if $T$ is global or an update transaction, $T$ is submitted to the certification protocol, at which point $T$ enters the Submitted state. The goal of the certification protocol is twofold. First, it propagates T's updates to $W R e p l i c a s(T)$. Second, it ensures that the execution of transactions is one-copy serializable. To submit $T$, sites use one of the certification protocols presented in Section IV.

- Submitted: To ensure one-copy serializability, the certification protocol checks whether $T$ observed an up-todate view of the database despite optimistic reads and concurrent updates. If $T$ passes the certification test, $T$ enters the Committed state; otherwise, $T$ passes to the Aborted state.

- Committed/Aborted: If $T$ commits, all sites in WReplicas $(T)$ apply its updates. Whatever T's outcome, $\operatorname{Proxy}(T)$ is notified.

In P-Store, local deadlocks are handled by the key-value store, and inter-site deadlocks cannot occur because a read operation releases its lock once the operation is completed and writes are ordered by atomic multicast.

When combined with any of the two certification protocols proposed in this paper, P-Store ensures genuine partial replication and one-copy serializability. Moreover, the following two liveness properties are also ensured:
- non-trivial certification: If there is a time after which no two read-write conflicting transactions are submitted, then eventually transactions are not aborted by certification

- termination: For every submitted transaction $T$, if $\operatorname{Proxy}(T)$ is correct, then all correct sites in WReplicas $(T)$ either commit or abort $T$.

\section{CERTIFying Transactions}

In this section, we present two certification protocols. The first one is simple but suffers from a convoy effect, that is, transaction certification may be delayed by transactions currently being certified. The second protocol seeks to minimize this undesired phenomenon.

\section{A. A Genuine Protocol}

The algorithm $\mathcal{A}_{g e}$ we present next relies on atomic multicast to certify transactions. We first present an overview of the algorithm and then present $\mathcal{A}_{g e}$ in detail.

Algorithm Overview: When a transaction $T$ is submitted for certification, Algorithm $\mathcal{A}_{g e}$ atomically multicasts $T$ to all groups storing keys read or updated by $T$. Upon ADelivering $T$, each site $s_{r}$ that replicates data items read by $T$ checks whether the values read are still up-to-date. To do so, $s_{r}$ compares the version of the data items read by $T$ against the versions currently stored in the database. If they are the same, $T$ passes certification at $s_{r}$, otherwise $T$ fails certification at $s_{r}$.

In a partially replicated context, $s_{r}$ may only store a subset of the keys read by $T$, in which case $s_{r}$ does not have enough information to decide on T's outcome. Hence, to satisfy nontrivial certification, we introduce a voting phase where sites replicating data items read by $T$ send the result of their certification test to each site $s_{w}$ in WReplicas $(T) .{ }^{1}$ Site $s_{w}$ can safely decide on $T$ 's outcome when $s_{w}$ received votes from a voting quorum for $T$. Intuitively, a voting quorum $V Q$ for $T$ is a set of sites such that for each data item read by $T$, there is at least one site in $V Q$ replicating this item. More formally, a quorum of sites is a voting quorum for $T$ if it belongs to $V Q(T)$, defined as follows:

$$
V Q(T)=\left\{V Q \mid V Q \subseteq \Pi \wedge T . r s \subseteq \bigcup_{s_{r} \in V Q} \operatorname{Items}\left(s_{r}\right)\right\}
$$

Transaction $T$ can safely commit when every site in a voting quorum for $T$ voted yes. If a site in the quorum votes $n o$, it means that $T$ read an old value and should be aborted to ensure serializability of the execution.

Figure 1 illustrates the execution of a global transaction $T$ that reads data items from groups $g_{1}$ and $g_{2}$. After all read requests have been executed, $T$ is submitted to $\mathcal{A}_{g e}$ for certification.

\footnotetext{
${ }^{1} \mathrm{~A}$ similar voting phase appears in [14]. In contrast to $\mathcal{A}_{g e}$, the protocol in [14] is non-genuine and relies on a total order to certify transactions.
} 


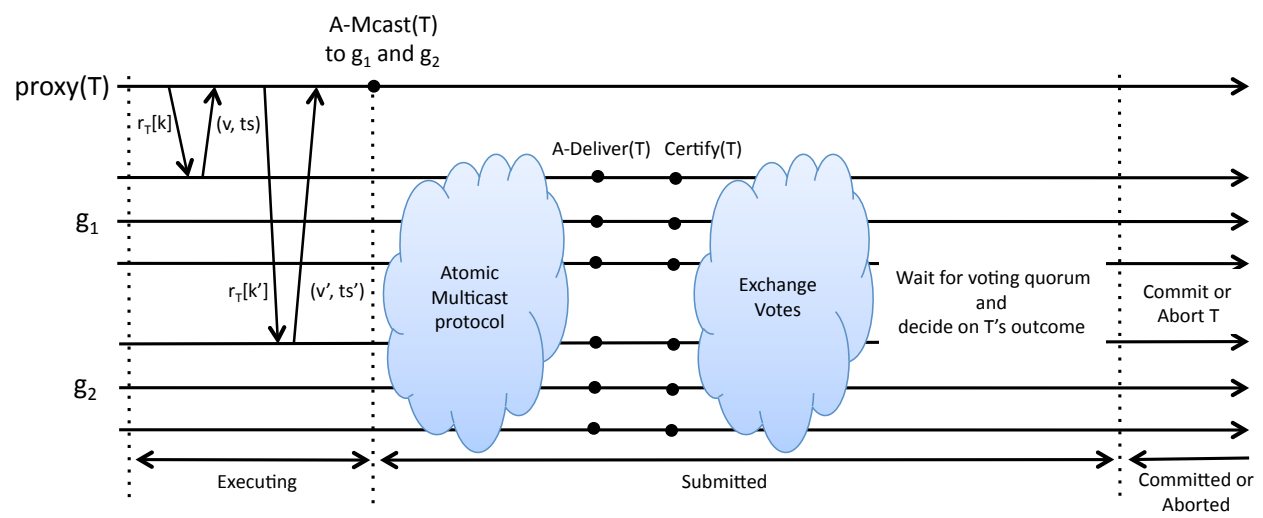

Figure 1. The execution and certification of a global transaction $T$ involving groups $g_{1}$ and $g_{2}$ with Algorithm $\mathcal{A}_{g e}$.

Algorithm in Detail: Algorithm $\mathcal{A}_{g e}$ (see next page) is composed of three concurrent tasks. Each line of the algorithm is executed atomically. The algorithm uses a global variable named Votes that stores the votes received, i.e., the results of the certification tests.

When a transaction $T$ is submitted, $\operatorname{Proxy}(T)$ atomically multicasts $T$ to $\operatorname{Replicas}(T)$ (line 10). Upon A-delivering $T$, each site $s$ that stores data items read by $T$ certifies $T$ (line 8). If $T$ is local, $s$ knows $T$ 's outcome at this point and, in case $T$ commits, $s$ applies $T$ 's updates to the database (lines 16-17). Otherwise, $T$ aborts (line 18). If $T$ is global, the result of the certification test is stored locally at $s$ and sent to every site $s_{w}$ in WReplicas $(T)$, except to members of $s$ 's group (lines 20-22). Each site $s_{w}$ waits until it receives votes from a voting quorum for $T$ at which point $s_{w}$ can safely decide on $T$ 's outcome (lines 23-24), and $T$ is handled similarly as local transactions (lines 25-28). The outcome of $T$ is then sent to $\operatorname{Proxy}(T)$ (line 29).

\section{B. Minimizing the Convoy Effect}

The convoy effect occurs when the certification of a transaction $T_{1}$ is delayed by another global transaction $T_{2}$ although $T_{1}$ is ready to be certified. In the certification proto$\operatorname{col} \mathcal{A}_{g e}$, this phenomenon may happen as follows: $T_{1}$ was ADelivered but it must wait until $T_{2}$ 's votes are received to be certified. As the frequency of submitted global transactions increases, this phenomenon deteriorates the performance of $\mathcal{A}_{g e}$ : an ever growing chain of transactions waiting to be certified is formed since only one global transaction can be certified per inter-group delay. We address this problem in Algorithm $\mathcal{A}_{g e}^{*}$. We first give an overview of the algorithm and then present $\mathcal{A}_{g e}^{*}$ in detail.

Algorithm Overview: To reduce the convoy effect, we seek to certify transactions in parallel as much as possible. In the scenario described above, this allows $T_{1}$ to be certified while $T_{2}$ 's votes are exchanged.

Obviously, not all transactions can be certified concurrently. Consider two read-write conflicting transactions $T_{0}$

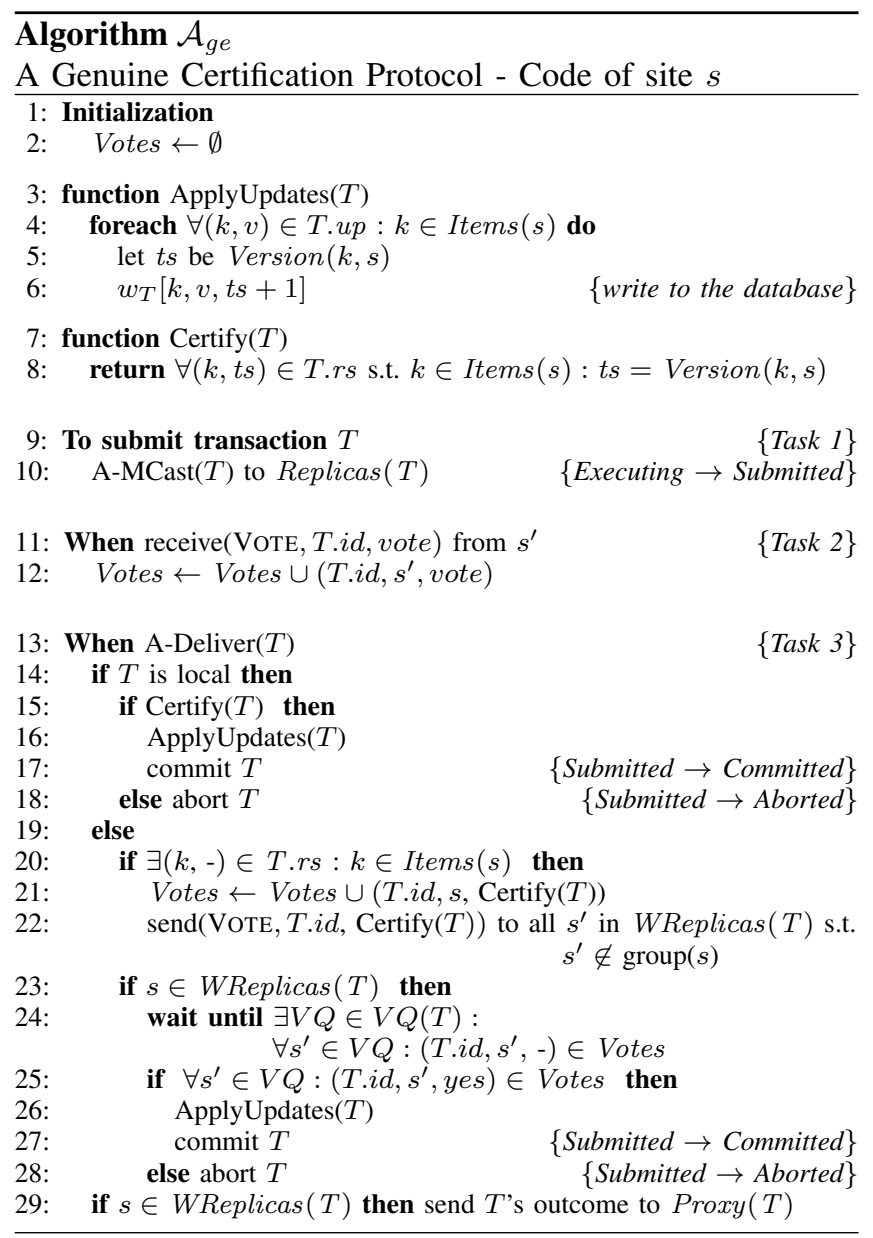

and $T_{1}$ such that $T_{i}$ reads key $k_{i}$ and writes key $k_{1-i}$ with $i \in[0,1]$. Further, suppose that keys $k_{0}$ and $k_{1}$ are replicated in different groups and thus a vote phase is needed to certify these transactions. If $T_{0}$ and $T_{1}$ were certified in parallel, a site $s_{1}$ could certify $T_{1}$ followed by $T_{2}$ while another site $s_{2}$ could certify $T_{2}$ before $T_{1}$. In this scenario, $s_{1}$ would vote 
"commit" for $T_{0}$ but "abort" for $T_{1}$, the inverse of what $s_{2}$ would do.

We observe that when transactions do not read-write conflict, their certification order does not matter since they do not affect each other. These transactions can thus be certified in parallel.

Nevertheless, the updates of such transactions must be applied in the order defined by atomic multicast. In short, this is because local read-only transactions are not certified. To illustrate this, consider the following execution that violates 1-SR. Suppose that two transactions $T_{1}$ and $T_{2}$ update keys $k_{1}$ and $k_{2}$, respectively, and on site $s_{1}, T_{1}$ commits before $T_{2}$, while on site $s_{2}, T_{2}$ commits before $T_{1}$. Consider in addition that on $s_{1}$ a local read-only transaction $T_{3}$ reads keys $k_{1}$ and $k_{2}$ before $T_{2}$ commits but after $T_{1}$ does, and that $s_{2}$ executes a local read-only transaction $T_{4}$ that reads the same data items as $T_{3}$ before $T_{1}$ commits but after $T_{2}$ does. This execution is not 1-SR: in a one-copy serial execution, $T_{3}$ must be placed before $T_{2}$ but after $T_{1}$. However, $T_{4}$ should be placed before $T_{1}$ but after $T_{2}$, which is impossible.

Algorithm in Detail: Algorithm $\mathcal{A}_{g e}^{*}$ is composed of four concurrent tasks. Each line of the algorithm is executed atomically. The algorithm uses two global variables: Votes stores the results of the certification tests, as in $\mathcal{A}_{g e}$, and Certify $Q$ is a FIFO queue of transactions that are being certified.

To submit a transaction $T, \operatorname{Proxy}(T)$ atomically multicasts $T$ to Replicas $(T)$ (line 11). When $T$ is A-Delivered at a site $s$ and $T$ does not read-write conflict with any transaction currently being certified, $s$ stores and sends the result of $T$ 's certification test if $s$ replicates data items read by $T$ and $T$ is global (lines 14-17). If $s$ is concerned by $T$ 's outcome, $s$ adds $T$ to the tail of Certify $Q$ (line 18).

Site $s$ then waits until there exists a transaction $T$ in Certify $Q$ whose outcome is known, i.e., Outcome $(T) \neq \perp$. Recall that $T$ 's outcome is known after $s$ certifies $T$ if $T$ is local (lines 4-5); if $T$ is global, its outcome is determined by votes from a voting quorum for $T$ (lines 6-7). If $T$ can commit, $s$ waits until $T$ is at the head of the certification queue before applying $T$ 's updates and committing $T$ (lines 19-22). This ensures that transaction updates are applied in the order defined by atomic multicast. If $T$ failed the certification test, $T$ can be aborted regardless of $T$ 's position in Certify $Q$ (lines 19). Transaction $T$ is then removed from Certify $Q$ and its outcome sent to $\operatorname{Proxy}(T)$ (lines 25-26).

\section{Why Does it Work?}

We briefly argue why P-Store ensures one-copy serializability, and refer the reader to [6] for a complete proof. We consider only certification protocol $\mathcal{A}_{g e}^{*}$ since $\mathcal{A}_{g e}$ is a special case of $\mathcal{A}_{g e}^{*}$.

Let $H$ be a replicated data history consisting of committed transactions only. History $H$ is $1-\mathrm{SR}$ iff $H$ is view-equivalent

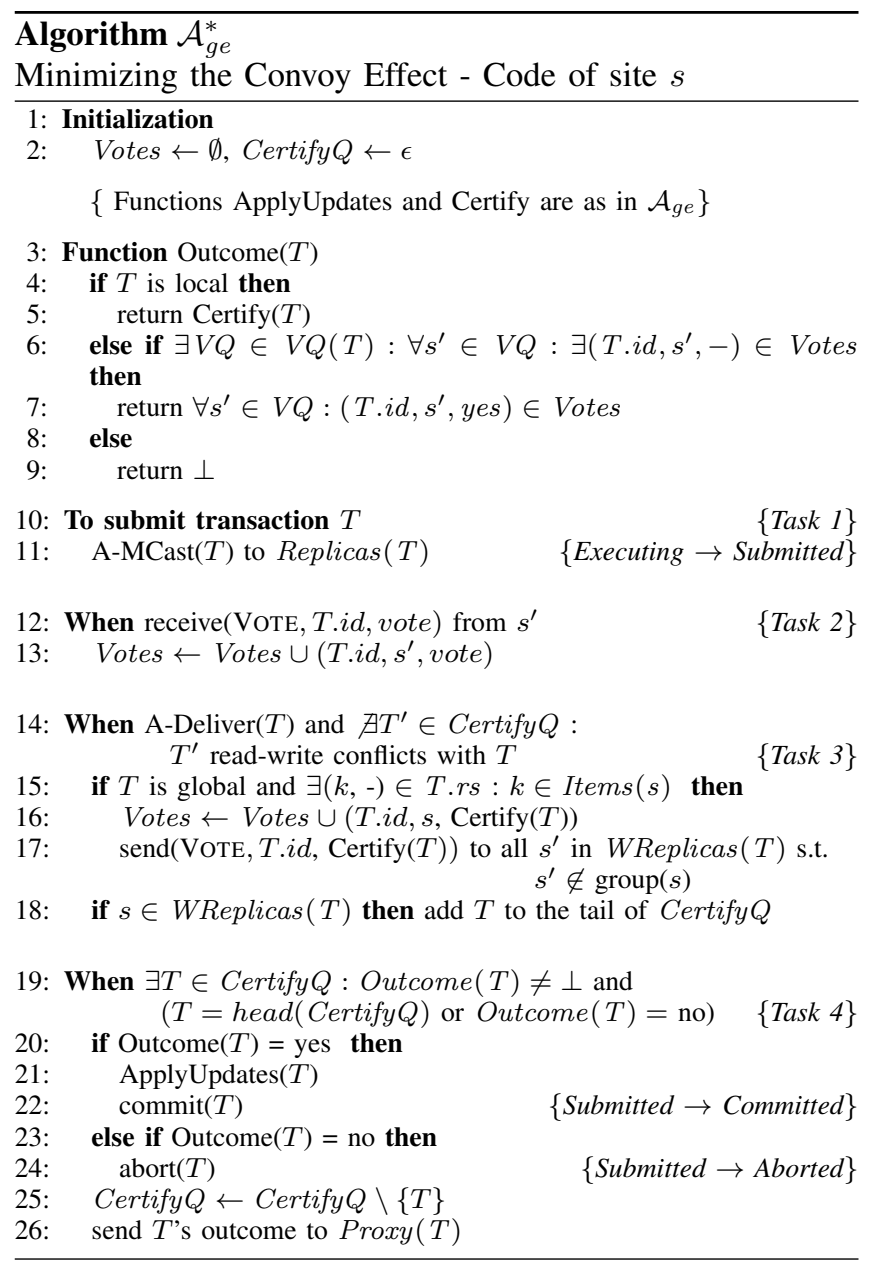

to some one-copy serial history $1 H$, where $H$ and $1 H$ are view-equivalent iff the following holds [10]:

1) $H$ and $1 H$ are defined over the same set of transactions,

2) $H$ and $1 H$ have the same read-from relationships on data items: $\forall T_{i}, T_{j} \in H$ (and hence, $T_{i}, T_{j} \in 1 H$ ): $T_{j}$ reads-x-from $T_{i}$ in $H$ iff $T_{j}$ reads-x-from $T_{i}$ in $1 H$, and,

3) For each final write $w_{T}[k, v, t s]$ in $1 H, w_{T}\left[k_{a}, v, t s\right]$ is also a final write in $H$ for some copy $k_{a}$ of key $k$.

We show how to construct a one-copy serial history $1 H$ that is view-equivalent to $H$. History $1 H$ consists of the same committed transactions as $H$, write operations follow the order defined by atomic multicast, and operations from different transactions do not interleave. In certification protocol $\mathcal{A}_{g e}^{*}$, a transaction $T$ passes the certification test iff the data items read by $T$ are still up-to-date. Hence, if $T$ commits then no transactions updated data items read by $T$ in the meantime. Consequently, $T$ reads a key $k$ written by some transaction $T^{\prime}$ in $H$ iff $T$ reads key $k$ from $T^{\prime}$ in $1 H$. The fact that $\mathcal{A}_{g e}^{*}$ certifies transactions that do not readwrite conflict in parallel does not matter since certifying them sequentially would produce the same result. Finally, 


\begin{tabular}{|c|c|c|c|c|c|}
\hline Algorithm & Genuine? & $\begin{array}{c}\text { Execution } \\
\text { latency }\end{array}$ & $\begin{array}{c}\text { Certification } \\
\text { latency }\end{array}$ & $\begin{array}{c}\text { Consistency } \\
\text { criterion }\end{array}$ \\
\hline$[3]$ & no & $\left(r_{r}+w_{r}\right) \times 2 \Delta$ & $2 \Delta$ & $O\left(n^{2}\right)$ \\
\hline$[5]$ & no & $\left(r_{r}+w_{r}+1\right) \times 2 \Delta$ & $2 \Delta$ & $O\left(n^{2}\right)$ \\
\hline$[4]$ & no & $\left(r_{r}+w_{r}\right) \times 2 \Delta$ & $2 \Delta$ & $O\left(k d^{2}\right)$ & 1 -copy-SI \\
\hline$[1]$ & yes & $r_{r} \times 2 \Delta \mid r_{r} \times 3 \Delta$ & $4 \Delta$ & $O\left(k^{2} d^{2}\right) \mid O\left(\left(r_{r}+k^{2}\right) \times d^{2}\right)$ & 1 -copy-SI \\
\hline$[2]$ & yes & $r_{r} \times 2 \Delta$ & $2 \Delta \mid 4 \Delta$ & $O\left(k^{2} d^{2}\right) \mid O\left(\left(r_{r}+w_{r}+k^{2}\right) \times d^{2}\right)$ & 1 -SR \\
\hline $\mathcal{A}_{g e} \& \mathcal{A}_{g e}^{*}$ & yes & $r_{r} \times 2 \Delta$ & $3 \Delta$ & $O\left(k^{2} d^{2}\right)$ \\
\hline
\end{tabular}

Table I

COMPARISON OF THE DATABASE REPLICATION PROTOCOLS $\left(r_{r}\right.$ AND $w_{r}$ ARE THE NUMBER OF REMOTE READS AND WRITES RESPECTIVELY, $n$ IS THE NUMBER OF SITES IN THE SYSTEM, $d$ IS THE NUMBER OF SITES PER GROUP, AND $k$ IS THE NUMBER OF GROUPS ADDRESSED BY $T$ ).

since writes to every key $k$ are ordered by atomic multicast, it follows directly that if $w_{T}[k, v, t s]$ is a final write to $k$ in $1 H$, then $w_{T}\left[k_{a}, v, t s\right]$ is also a final write to $k$ in $H$.

\section{RELATED WORK}

Numerous protocols for full database replication have been proposed [15], [16], [17], [18], some of which have been evaluated in wide area networks [19]. Fewer protocols for partial replication exist. These protocols can be grouped in two categories: those that are optimized for local area networks [20], [21], [22], [14], and those that are topologyoblivious. In the following, we review protocols from the second category that either provide a generalized form of snapshot isolation (1-copy-SI) [3], [5], [4] or one-copy serializability (1-SR) [1], [2].

With 1-copy-SI, transactions read data from a possibly old committed snapshot of the database and execute without interfering with each other. A transaction $T$ can only successfully commit if no other transaction $T^{\prime}$ updated the same data items and committed after $T$ started (first-committerwins rule). This consistency criterion never blocks nor aborts read-only transactions and update transactions are never blocked nor aborted due to read-only transactions.

To the best of our knowledge, none of the protocols that ensure 1-copy-SI guarantee genuine partial replication. In fact, to certify a transaction $T$, either $T$ is atomically multicast to all sites in the system [3], [5], or $T$ is sent to a certifier group [4]. Although the latter protocol reduces the communication overhead, messages must now be exchanged over wide area links for local transactions. Further, the certifier group may become the bottleneck under high loads since it must certify all transactions. To guarantee that $T$ observes a consistent view of the database when $T$ is global, the protocols in [3], [4] take dummy snapshots locally after each transaction commit. In [5], this is achieved by atomically multicasting an additional snapshot message at the beginning of $T$ 's execution.

In [1] the authors propose a database replication protocol based on atomic multicast that ensures 1-SR. Every read operation on data item $x$ is multicast to the group replicating $x$; writes are multicast along with the commit request. The delivered operations are executed on the replicas using strict two-phase locking and results are sent back to the client. A final atomic commit protocol ensures transaction atomicity. In the atomic commit protocol, every group replicating a data item read or written by a transaction $T$ sends its vote to a coordinator group, which collects the votes and sends the result back to all participating groups.

In [2], a protocol that allows transactions to be executed on multiple sites is presented. To certify a transaction $T$, $T$ is reliably multicast to Replicas $(T)^{2}$, and each operation of $T$ on some data item $x$ is atomically multicast to the replicas of $x$. Sites then build the graph $G$ of transactions that precede $T$ in the execution by exchanging their partial view of $G$. One-copy serializability is ensured by checking that $G$ is acyclic. These last two operations, namely building $G$ and checking that $G$ is acyclic, can be expensive.

Two algorithms based on atomic multicast that ensure genuine partial replication are presented in this paper. They require a single atomic multicast per transaction plus a vote phase, in case the transaction is global. Transactions may execute at multiple sites to allow a flexible partitioning of the database even if transactions access the database in its entirety. Algorithm $\mathcal{A}_{g e}$ may suffer from the convoy effect since it certifies transactions sequentially. The second algorithm $\mathcal{A}_{g e}^{*}$ alleviates this undesired phenomenon by allowing non-conflicting transactions to be certified in parallel.

Table I compares the properties and cost of the reviewed protocols. Column two indicates whether the protocols ensure genuine partial replication. The subsequent three columns present the cost of the protocols, namely the intergroup latency to execute and certify a transaction $T$, and the total number of inter-group messages exchanged to execute and certify $T$. To compute these costs, we consider that $T$ is global and is executed from within some group $g$. Transaction $T$ issues $r_{r}$ remote reads and $w_{r}$ remote writes. These operations access data items stored outside of $g$ and thus require inter-group communication. Both $r_{r}$ and $w_{r}$ are in the order of $k$, the number of groups addressed by $T$. Further, we assume that groups are correct, neither failures nor failure suspicions occur, inter-group messages have a

\footnotetext{
${ }^{2}$ Reliable multicast ensures all properties of atomic multicast except uniform prefix and acyclic order.
} 
delay of $\Delta$, and intra-group message delays are assumed to be negligible. In Table I, costs are computed by using the atomic multicast algorithm in [23]. This protocol has a latency of $\Delta$ and $2 \Delta$ for messages addressed to one and multiple groups respectively, and sends $O\left(x^{2}\right)$ intergroup messages to deliver multicast messages, where $x$ is the number of sites to which the transaction is multicast. In columns four, five, and six, we report the costs of the algorithms when data items are replicated in one and two groups respectively. When these costs are identical, we report a single value.

\section{THE IMPLEMENTATION OF P-STORE}

We implemented P-Store in Java on top of BerkeleyDB (BDB). To execute a transaction $T$, a client sends read and write requests to $\operatorname{Proxy}(T)$, one of the sites in Replicas $(T)$. Write requests are buffered by $\operatorname{Proxy}(T)$ and each read of key $k$ is executed at some site that stores $k$ inside a BDB transaction. When $T$ is ready to commit, $\operatorname{Proxy}(T)$ submits $T$ along with $T$ 's set of key-version pairs read and $T$ 's updates using one of the certification protocols presented in this paper. If $T$ passes the certification test, a BDB transaction applies $T$ 's updates to the database in the order defined by atomic multicast. Otherwise, P-Store re-executes $T$ and resubmits $T$ to the certification protocol.

Certification protocols $\mathcal{A}_{g e}$ and $\mathcal{A}_{g e}^{*}$ are implemented on top of a genuine atomic multicast protocol optimized for wide area networks [23]. Performance evaluations of this protocol can be found in [24]. When a transaction $T$ is ADelivered at some site $s, s$ assigns a certifier thread to $T$. This thread executes the certification test for $T$ and applies $T$ 's update to the database as soon as $T$ does not read-write conflict with transactions currently being certified. Certifiers are part of a thread pool whose size is configurable. When P-Store uses $\mathcal{A}_{g e}$ to certify transactions, the certifier pool contains a single thread.

\section{PERformance Evaluation}

In this section, we present an experimental evaluation of the performance of P-Store with the certification protocols $\mathcal{A}_{g e}$ and $\mathcal{A}_{g e}^{*}$. We start by presenting the system settings and the benchmark used to assess the protocols. We then evaluate the impact of the convoy effect on $\mathcal{A}_{g e}$ and asses the scalability of P-Store when partial and full replication are assumed.

\section{A. Experimental Settings}

The system: The experiments were conducted in a cluster of 24 nodes connected with a gigabit switch. Each node is equipped with two dual-core AMD Opteron $2 \mathrm{Ghz}$, $4 \mathrm{~GB}$ of RAM, and runs Linux 2.6. In all experiments, each group consisted of 3 nodes, and the number of groups varied from 2 to 8 . We assumed that groups were correct and used an atomic multicast service optimized for this assumption. To provide higher degrees of resilience, it would have sufficed to replace the atomic multicast service with an implementation that tolerates group crashes [12] and replicate data items in multiple groups. Note, however, that this would come at a cost. The bandwidth and message delay of our local network, measured using netperf and ping, were about $940 \mathrm{Mbps}$ and $0.05 \mathrm{~ms}$ respectively. To emulate inter-group delays with higher latency and lower bandwidth, we used the Linux traffic shaping tools. We considered a network in which the message delay between any two groups follows a normal distribution with a mean of $50 \mathrm{~ms}$ and a standard deviation of $5 \mathrm{~ms}$, and each group is connected to the other groups via a $1.25 \mathrm{MBps}(10 \mathrm{Mbps})$ full-duplex link.

BerkeleyDB was configured with asynchronous disk writes and logging in memory. Moreover, on each site $s$, the cache was big enough to hold the entire portion of the database replicated at $s$. This corresponds to a setting were data durability is ensured through replication.

The benchmark: We measured the performance of the protocols using a modified version of the industry standard TPC-B benchmark [25]. TPC-B consists of update transactions only, and defines one transaction type that deposits (or withdraws) money from an account. In our implementation of TPC-B, each transaction reads and updates three data items: the account, the teller in charge of the transaction, and the branch in which the account resides. Accounts and tellers are associated with a unique branch.

We horizontally partitioned the branch table such that each group is responsible for an equal share of the data. Accounts and tellers of a branch $b$ were replicated in the same group as $b$, and members of a given group replicated the same set of data items. Before partitioning, the database consisted of 3'600 branches, 36'000 tellers, and 360'000 accounts.

In TPC-B, about $15 \%$ of transactions involve two groups, that is, the teller in charge of the transaction is replicated in a different group than the group in which the branch and account are stored. The remaining $85 \%$ of transactions access data in the same group. To assess the scalability of our protocols, we parameterized the benchmark to control the proportion $p$ of global transactions. In the experiments, we report measurements when $p$ varies from $0 \%$ to $50 \%$.

Each node of the system contained an equal number of clients that executed the benchmark in a closed loop: each client executed a transaction $T$ and waited until it was notified of $T$ 's outcome before executing the next one. Each client $c$ was placed in the same group as the teller in charge of handling $c$ 's transactions, and inside each group $g$ the load generated by $g$ 's clients was equally shared among $g$ 's replicas. For all the experiments, we report the average transaction execution time (in milliseconds) as a function of the throughput, i.e., the number of transactions committed per second. We computed $95 \%$ confidence intervals for the transaction execution times but we do not report them here as they were always smaller than $5 \%$ of the average execution 
time. The throughput was increased by adding an equal number of clients to each node of the system and, on average, four hundred thousand transactions were executed per experiment. In steady state, that is, when the system supports the client load, the throughput of committed transactions equals the input load minus the rate of aborted transactions. Unless we explicitly state otherwise, the executions were cpu-bound at peak loads.

\section{B. Assessing the Convoy Effect}

We explore the influence of the number of certifier threads on the performance of P-Store. We consider a system with four groups, one percent of global transactions, and vary the number of certifier threads from one to one hundred fifty. With any pool size bigger than one, the certification protocol used is $\mathcal{A}_{g e}^{*}$, otherwise it is $\mathcal{A}_{g e}$.

In Figure 2, we observe that the convoy effect affects both latency and throughput despite a low percentage of global transactions. With one certifier thread, as soon as sites certify a global transaction, no other transactions can be certified for the duration of the vote exchange, that is, one inter-group delay. This creates long chains of transactions waiting to be certified and limits throughput. Adding extra certifiers quickly improves the performance of P-Store which reaches its peak bandwidth with one hundred fifty threads. With this pool size, the peak throughput reached by P-Store more than doubled compared to when P-Store uses a single certifier. We observed that the difference between these two certification protocols grows when the percentage of global transactions increases.

In the following experiments, we configured $\mathcal{A}_{g e}^{*}$ to use one hundred certifiers since the performance gained by adding an extra fifty certifiers is small.

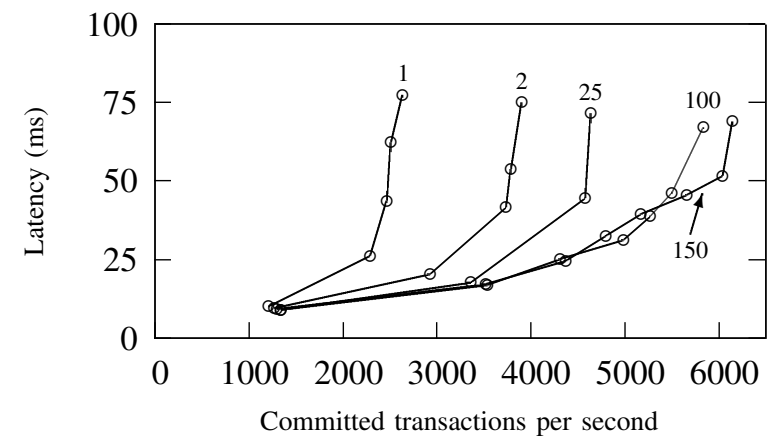

Figure 2. The influence of the number of certifier threads in a system with four groups and $1 \%$ of global transactions.

\section{Full versus Partial Replication}

In the following, we assess the scalability of P-Store and compare certification protocol $\mathcal{A}_{g e}^{*}$ against a certification protocol denoted as $\mathcal{A}_{\text {full }}$ that assumes full replication, i.e., all sites store the entire database.
Protocol $\mathcal{A}_{\text {full }}$ is based on $\mathcal{A}_{g e}^{*}$ : non-conflicting transactions are certified in parallel and updates are applied in the order defined by atomic multicast. When an update transaction $T$ is submitted to $\mathcal{A}_{\text {full }}, T$ is atomically multicast to all sites to be certified and to propagate its updates. Since full replication is assumed, all transactions are local and do not require a vote phase. The certifier pool of $\mathcal{A}_{\text {full }}$ contains one hundred threads.

We note that $\mathcal{A}_{g e}^{*}$ and $\mathcal{A}_{\text {full }}$ use different atomic multicast protocols: $\mathcal{A}_{g e}^{*}$ uses a genuine atomic multicast that requires two inter-group delays to deliver a multicast message if the message is addressed to multiple groups, and none when the message is addressed to a single group and the sender is a member of that group. To multicast a message to all sites, $\mathcal{A}_{\text {full }}$ relies on a non-genuine multicast protocol that needs a single inter-group delay [24].

We compare the performance of $\mathcal{A}_{\text {ge }}^{*}$ and $\mathcal{A}_{\text {full }}$ in a setting where the database is split into as many partitions as there are groups, and each group replicates $x$ partitions. We denote $x$ as the replication factor or number of copies. With $x$ equal to one, the data is perfectly partitioned such that no two groups replicate the same data item-this is the setting $\mathcal{A}_{g e}^{*}$ used up to now. With $x$ equal to the number of groups, we fall back to full replication, i.e., $\mathcal{A}_{\text {full }}$.

Local Replication: We start the comparison between $\mathcal{A}_{\text {full }}$ and $\mathcal{A}_{\text {ge }}^{*}$ with a replication factor of one. We assess the scalability of the protocols by varying the number of groups and percentage of global transactions.

In Figure 3(a) and 3(b), we report the latency of P-Store as a function of throughput when using certification protocols $\mathcal{A}_{\text {ge }}^{*}$ and $\mathcal{A}_{\text {full }}$. Figure 3(a) presents the average latency of the certification protocols considering both local and global transactions; Figure 3(b) plots the latency of global and local transactions separately. We consider from $0 \%$ to $50 \%$ of global transactions and a system with four groups. $\mathcal{A}_{\text {ge }}^{*}$ presents a similar latency as $\mathcal{A}_{\text {full }}$ with $25 \%$ of global transactions but supports higher loads (see Figure 3(a)). Any percentage of global transactions higher than $25 \%$ makes full replication more attractive than partial replication. This is explained by the extra cost paid by $\mathcal{A}_{g e}^{*}$ to fetch remote data items and execute vote phases to handle global transactions.

With lower percentages of global transactions, $\mathcal{A}_{g e}^{*}$ provides lower latencies and improves the peak throughput of $\mathcal{A}_{\text {full }}$ by a factor of $2.1,2.7,3.7$, and 6.3 when the percentage of global transactions is respectively $15 \%, 5 \%$, $1 \%$, and $0 \%$. When no transactions are global, each group acts as a completely independent replicated system, the corresponding curve in Figure 3(a) thus only serves as an illustrative purpose.

In Figure 3(b), we observe that local transactions are executed faster with $\mathcal{A}_{g e}^{*}$ than with $\mathcal{A}_{f u l l}$ : with the latter certification protocol, transactions are multicast to all groups as opposed to only the local group with $\mathcal{A}_{g e}^{*}$. At low loads, global transactions require about 300 milliseconds to be 


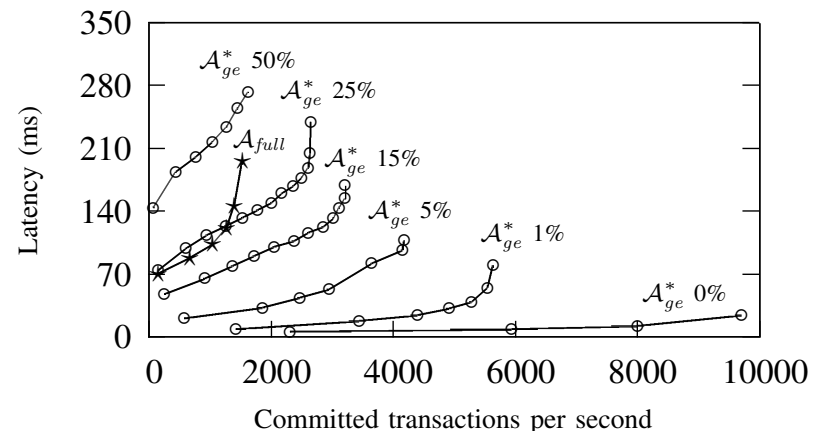

(a) 4 groups, varying the percentage of global transactions

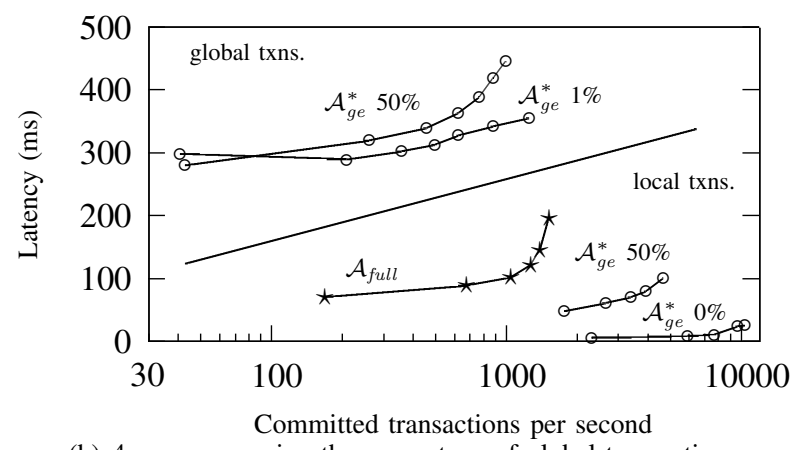

(b) 4 groups, varying the percentage of global transactions

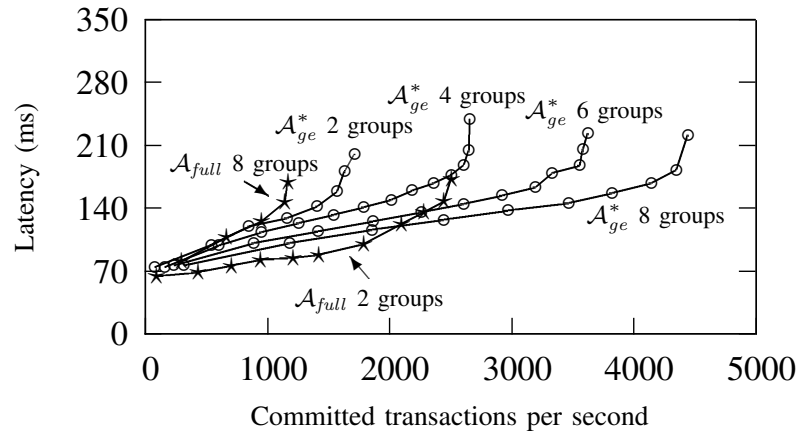

(c) $25 \%$ of global transactions, varying the number of groups

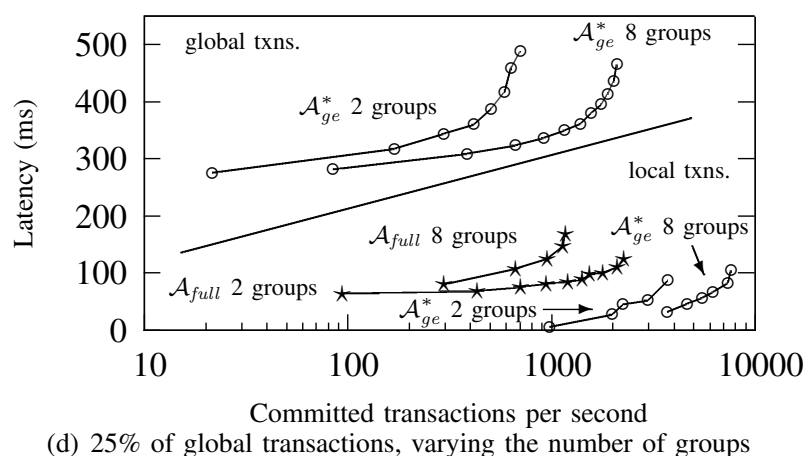

(d) $25 \%$ of global transactions, varying the number of groups

Figure 3. The scalability of $\mathcal{A}_{\text {full }}$ and $\mathcal{A}_{\text {ge }}^{*}$ when the percentage of global transactions and the number of groups vary.

executed by P-Store and certified with $\mathcal{A}_{g e}^{*}$ : two inter-group delays are needed to fetch remote data items, two delays are required to atomically multicast the transaction, and the last inter-group delay allows to carry out the vote phase. In total, inter-group communication takes 250 milliseconds, the remaining 50 milliseconds are due to intra-group communication and processing overhead. Note that with $\mathcal{A}_{\text {full }}$, all transactions are local since sites store the entire database.

In Figure 3(c) and 3(d), we study the scalability of $\mathcal{A}_{g e}^{*}$ and $\mathcal{A}_{\text {full }}$ when the number of groups varies and consider $25 \%$ of global transactions. As before, Figure 3(c) presents the average latency of the protocols when considering both local and global transactions and Figure 3(d) separates local and global transactions. $\mathcal{A}_{\text {full }}$ does not scale when the number of groups increases. In fact, $\mathcal{A}_{\text {full }}$ performs best with two groups; with eight groups the execution is networkbound. In contrast, $\mathcal{A}_{g e}^{*}$ presents a scale-out of roughly 0.7 up to eight groups, that is, multiplying the number of groups by $k$ increases the peak throughput of $\mathcal{A}_{g e}^{*}$ by a factor of $0.7 k$. Moreover, $\mathcal{A}_{g e}^{*}$ with eight groups can support a load that is 1.7 times higher than the best peak throughput of $\mathcal{A}_{\text {full }}$ among all considered system sizes. This shows that $\mathcal{A}_{g e}^{*}$ offers good scalability even when $25 \%$ of the workload involves multiple groups. Figure 3(d) shows similar trends when comparing $\mathcal{A}_{g e}^{*}$ with $\mathcal{A}_{\text {full }}$.

We note that at peak loads, from $7 \%$ to $15 \%$ of trans- actions were aborted by $\mathcal{A}_{g e}^{*}-\mathcal{A}_{\text {full }}$ never aborted more than $5 \%$ of the transactions. In $\mathcal{A}_{g e}^{*}$, aborts were primarily caused by the optimistic reads of global transactions. We are currently working on techniques to reduce this phenomenon.

Replicating Data across Groups: To reduce the amount of data items fetched from remote groups and increase the locality of the execution, it may be interesting to replicate data items in multiple groups. To evaluate this idea, we consider a replication factor of two, that is, data items are replicated in two groups.

In Figures 4(a) and 4(b), we report results in a system with four groups and 100\% of updates. Figures 4(c) and 4(d) illustrate the performance of the protocols with $20 \%$ of updates-read-only transactions read a single account. Recall that update transactions also perform reads and may benefit from replicating data items in multiple groups to allow local reads.

Maintaining copies of each data item in two groups harms the latency and scalability of $\mathcal{A}_{g e}^{*}$ dramatically with $25 \%$ of global transactions (see Figure 4(a)). Transactions are now atomically multicast to twice as many groups to be certified. In particular, global transactions must be multicast to all four groups of the system. Interestingly, reducing the percentage of global transactions to $1 \%$ does not affect this result significantly-in fact when considering local and global transactions separately, this difference becomes 


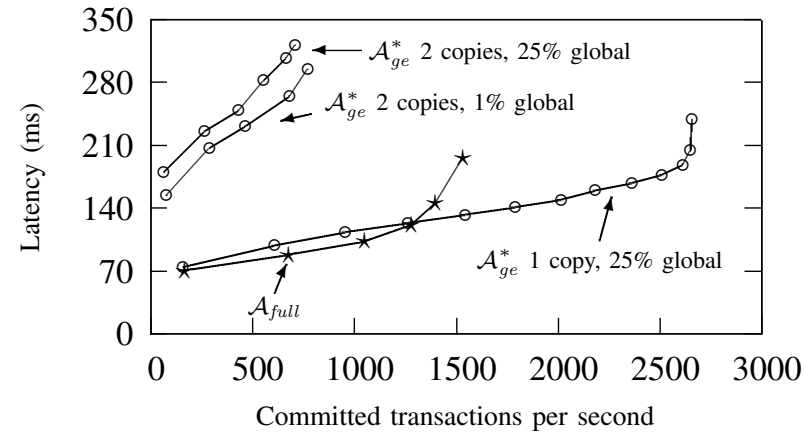

(a) 4 groups, $100 \%$ of updates, varying the replication factor

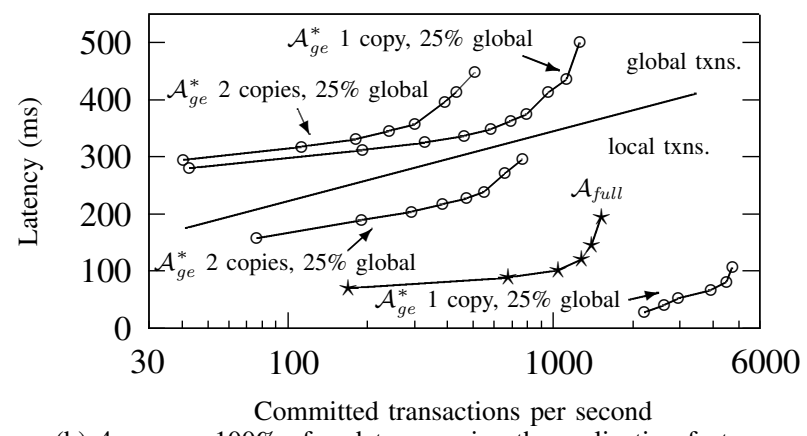

(b) 4 groups, $100 \%$ of updates, varying the replication factor

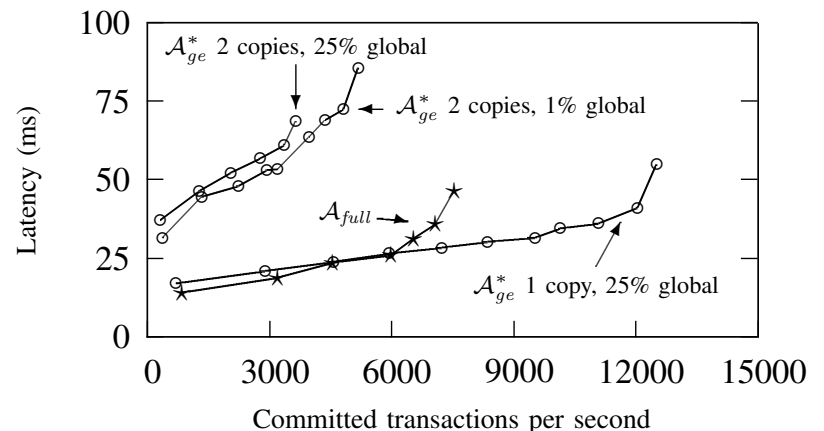

(c) 4 groups, $20 \%$ of updates, varying the replication factor

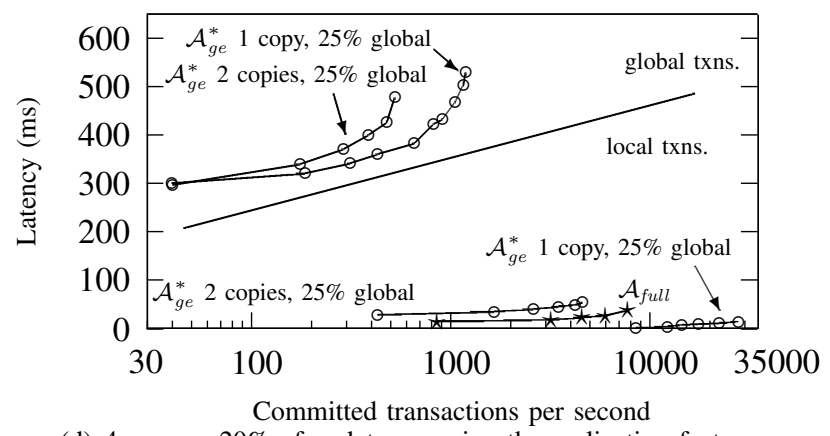

(d) 4 groups, $20 \%$ of updates, varying the replication factor

Figure 4. The scalability of $\mathcal{A}_{\text {full }}$ and $\mathcal{A}_{g e}^{*}$ when the replication factor and the percentage of update transactions vary.

insignificant and we thus omit the curves for $1 \%$ of global transactions in Figure 4(b). With two copies, the updates of local transactions must be applied to two groups, compared to only one group otherwise. Hence local transactions now have a much higher latency than with a replication factor of one (see Figure 4(b)). Moreover, with two copies, $\mathcal{A}_{g e}^{*}$ requires roughly twice as much time as $\mathcal{A}_{\text {full }}$ to commit local transactions. This is because $\mathcal{A}_{g e}^{*}$ uses an atomic multicast protocol that requires twice the number of inter-group delays to deliver messages addressed to multiples groups, i.e., two for $\mathcal{A}_{g e}^{*}$ as opposed to one for $\mathcal{A}_{\text {full }}$ [24].

This situation does not change when the workload is composed of only $20 \%$ of updates (see Figures 4(c) and 4(d)). The only difference we observe is a significant decrease in the average latency of local transactions (see Figure 4(d)). This is because read-only transactions now constitute $80 \%$ of the workload and commit without inter-site communication. On average, these transactions require less than a millisecond to commit, regardless of the protocol and the load.

In Figures 4(a) and 4(c), $\mathcal{A}_{\text {full }}$ provides better performance than $\mathcal{A}_{g e}^{*}$ with two copies and regardless of the proportion of global transactions. This suggests that performing remote reads is less costly than replicating data across groups for the system size considered.

\section{Summary}

Based on the results above, we provide a tentative decision diagram to determine whether to deploy full or partial replication given the workload. Figure 5 advocates partial replication when the number of groups is important and global transactions access few groups. We suspect that the percentage of read-only transactions does not affect the decision procedure, except in special cases (e.g., when the majority of read-only transactions read data items from multiple groups). Further refining this decision procedure is future work.

\section{CONCLUSION}

P-Store is a partially replicated key-value store for wide area networks that allows transactions to optimistically execute at multiple sites and certifies transactions in a genuine manner: to certify a transaction $T$ only sites that replicate data items read or written by $T$ exchange messages. The certification protocol $\mathcal{A}_{g e}^{*}$ proposed in this paper allows to reduce the convoy effect by certifying non-conflicting transactions in parallel. To guarantee serializability of the transaction execution, P-Store makes a thrifty use of an atomic multicast service: a single message is atomically multicast during $T$ 's certification. In case $T$ is global, an extra vote phase is executed to ensure that sites agree on $T$ 's outcome. This is in contrast to previously proposed solutions 


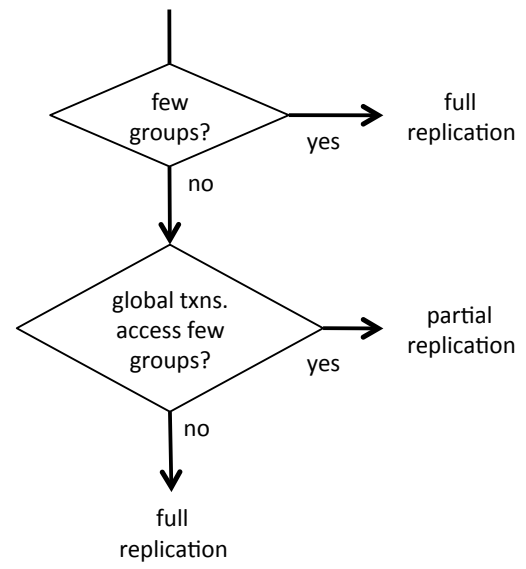

Figure 5. Deciding between full and partial replication.

that either invoke the atomic multicast service multiple times to handle each transaction or are non-genuine.

Our experimental results show that the certification proto$\operatorname{col} \mathcal{A}_{g e}^{*}$ effectively reduces the convoy effect and doubles the peak throughput of P-Store even when only one percent of transactions are global. We also observed that P-Store scales better than a fully replicated solution when the percentage of global transactions is no more than twenty-five percent and provides an almost linear scale-out up to at least eight groups.

As future work, we plan to further investigate the parameters that influence the scalability of partial replication and refine our decision procedure to determine when partial replication is a better choice than full replication.

\section{REFERENCES}

[1] U. Fritzke and P. Ingels, "Transactions on partially replicated data based on reliable and atomic multicasts." in Proceedings of ICDCS'01. IEEE Computer Society, pp. 284-291.

[2] P. Sutra and M. Shapiro, "Fault-tolerant partial replication in large-scale database systems," in Proceedings of Euro-Par'08, pp. 404-413.

[3] D. Serrano, M. Patiño Martínez, R. Jiménez-Peris, and B. Kemme, "Boosting database replication scalability through partial replication and 1-copy-snapshot-isolation," in Proceedings of PRDC '07. Washington, DC, USA: IEEE Computer Society, pp. 290-297.

[4] D. Serrano, M. Patiño-Martínez, R. Jiménez-Peris, and B. Kemme, "An autonomic approach for replication of internet-based services," in Proceedings of SRDS'08, 2008, pp. $127-136$.

[5] J. E. Armendáriz, A. Mauch-Goya, J. R. G. de Mendívil, and F. D. Muñoz, "Sipre: a partial database replication protocol with si replicas," in Proceedings of SAC '08. New York, NY, USA: ACM, pp. 2181-2185.

[6] N. Schiper, P. Sutra, and F. Pedone, "P-store: Genuine partial replication in wide area networks," University of Lugano, Tech. Rep. 2010/003, 2010.
[7] M. J. Fischer, N. A. Lynch, and M. S. Paterson, "Impossibility of distributed consensus with one faulty process," Journal of the $A C M$, vol. 32, no. 2, pp. 374-382, 1985.

[8] T. D. Chandra and S. Toueg, "Unreliable failure detectors for reliable distributed systems," Journal of the ACM, vol. 43, no. 2, pp. 225-267, 1996.

[9] C. Beeri, P. A. Bernstein, and N. Goodman, "A model for concurrency in nested transactions systems," J. $A C M$, vol. 36, no. 2, pp. 230-269, 1989.

[10] P. A. Bernstein, V. Hadzilacos, and N. Goodman, Concurrency Control and Recovery in Database Systems. AddisonWesley, 1987.

[11] U. Fritzke, P. Ingels, A. Mostéfaoui, and M. Raynal, "Faulttolerant total order multicast to asynchronous groups," in Proceedings of SRDS'98. IEEE Computer Society, pp. 578585.

[12] N. Schiper and F. Pedone, "Solving atomic multicast when groups crash," in Proceedings of OPODIS'08, pp. 481-495.

[13] R. Guerraoui and A. Schiper, "Genuine atomic multicast in asynchronous distributed systems," Theoretical Computer Science, vol. 254, no. 1-2, pp. 297-316, 2001.

[14] N. Schiper, R. Schmidt, and F. Pedone, "Optimistic algorithms for partial database replication," in In Proceedings of OPODIS'06. Springer, pp. 81-93.

[15] B. Kemme and G. Alonso, "Don't be lazy, be consistent: Postgres-r, a new way to implement database replication," in The VLDB Journal, 2000, pp. 134-143.

[16] M. Patiño-Martínez, R. Jiménez-Peris, B. Kemme, and G. Alonso, "Middle-r: Consistent database replication at the middleware level," ACM Trans. Comput. Syst., vol. 23, no. 4, pp. 375-423, 2005.

[17] Y. Lin, B. Kemme, M. Patiño Martínez, and R. JiménezPeris, "Middleware based data replication providing snapshot isolation," in Proceedings of SIGMOD '05. New York, NY, USA: ACM, pp. 419-430.

[18] F. Pedone and S. Frølund, "Pronto: High availability for standard off-the-shelf databases," Journal of Parallel and Distributed Computing, vol. 68, no. 2, pp. 150-164, 2008.

[19] Y. Lin, B. Kemme, M. Patiño-Martínez, and R. JiménezPeris, "Consistent data replication: Is it feasible in wans?" in Proceedings of Euro-Par'05, pp. 633-643.

[20] E. Cecchet, J. Marguerite, and W. Zwaenepoel, "C-jdbc: Flexible database clustering middleware," in USENIX Annual Technical Conference, FREENIX Track, 2004, pp. 9-18.

[21] A. Sousa, F. Pedone, R. Oliveira, and F. Moura, "Partial replication in the database state machine," in Proceedings of NCA'01. IEEE Computer Society, 2001, pp. 298-309.

[22] C. Coulon, E. Pacitti, and P. Valduriez, "Consistency management for partial replication in a high performance database cluster," in Proceedings of ICPADS'05, vol. 1. Los Alamitos, CA, USA: IEEE Computer Society, pp. 809-815.

[23] N. Schiper and F. Pedone, "On the inherent cost of atomic broadcast and multicast in wide area networks," in Proceedings of ICDCN'08. Springer, pp. 147-157.

[24] N. Schiper, P. Sutra, and F. Pedone, "Genuine versus nongenuine atomic multicast protocols for wide area networks: An empirical study," in Proceedings of SRDS'09. IEEE Computer Society, 2009, pp. 166-175.

[25] "Transaction processing performance council (tpc) - benchmark b," http://www.tpc.org/tpcb/. 